\title{
Fabrication of Mechanical Durable Polysiloxane Superhydrophobic Materials
}

\author{
Mengnan Qu, Jinmei He, Sun Zhe, Kanshe Li, Xiangrong Liu, and Chunxia Yu \\ College of Chemistry and Chemical Engineering, Xian University of Science and Technology, Xian 710054, China \\ Correspondence should be addressed to Jinmei He; jinmhe@xust.edu.cn
}

Received 23 November 2014; Accepted 13 March 2015

Academic Editor: Ion Tiginyanu

Copyright (C) 2015 Mengnan Qu et al. This is an open access article distributed under the Creative Commons Attribution License, which permits unrestricted use, distribution, and reproduction in any medium, provided the original work is properly cited.

\begin{abstract}
A mechanical durable polysiloxane superhydrophobic surface was successfully prepared by means of polymerization of silanes blending with particles. The as-prepared polysiloxane surface showed stable superhydrophobicity even after the surface underwent a long distance friction. The superhydrophobicity of the polysiloxane materials can be even slightly enhanced by the surface abrasion. The scanning electron microscopy demonstrated that the micro- and nanometer structures distributed through the whole materials thickness are responsible for the mechanical durable superhydrophobicity.
\end{abstract}

\section{Introduction}

The wettability of solid surface is one of the most important aspects in both theoretical and industrial applications [1]. One of the extreme cases of wettability, superhydrophobicity, has aroused considerable interests for many researchers inspired by the water-repellent nature of lotus leaves in recent years [2]. Heretofore, numerous methods were presented to fabricate the superhydrophobic surfaces with various materials by mimicking the surface of lotus leaves [3-10]. Due to its novel and important properties, the superhydrophobic surfaces were greatly anticipated to be used in applications such as self-cleaning coatings [11], anti-icing and fogging surfaces [12-14], nonwetting fabrics $[15,16]$, and buoyancy and flow enhancement $[17,18]$. However, there are still quite few real applications with superhydrophobic materials so far.

Durability is of great importance in many applications of superhydrophobic surface. The micro- and nanoscale surface topography, which is essential for superhydrophobicity, can be very easily destroyed by even a slight friction on the surface. Up to date, studies have begun to address the mechanical durability and methods were developed to enhance the durability of superhydrophobic surface [19], such as fabricating self-healing surfaces, enhancing the robustness of the hierarchical structure, and fabricating with hydrophobic materials. Li et al. demonstrated a self-healing superhydrophobic coating fabricated by preserving healing agents of reacted fluoroalkylsilane in layered polymeric coatings [20]. When the top layer of fluoroalkyl chains decomposes or the coatings are scratched, the healing agents migrate to the surface to restore the superhydrophobicity. In this case, it takes special conditions and hour's time to restore the superhydrophobicity and the fluoroalkylsilane reagent is still required. It still remains a great challenge to fabricate superhydrophobic materials with excellent antiwear property.

In the current study, we presented a facile and cheap method to fabricate wear-durable superhydrophobic materials. Inspired by the ability that the lotus leaf can regenerate its microstructures, we have presented a strategy to fabricate the materials which are made up of the microstructures through the whole thickness of the materials to resist the surface abrasion. When the initial surface suffered abrasion, a new surface with new hierarchical roughness will appear. Herein, the micro- and nanoparticles were used to shape the hierarchical roughness and the organic silanes were used to fix these particles. The as-prepared polysiloxane superhydrophobic materials showed good antiwear property. From both theoretical and practical aspects, the present results and the fabrication strategy are of great significance 


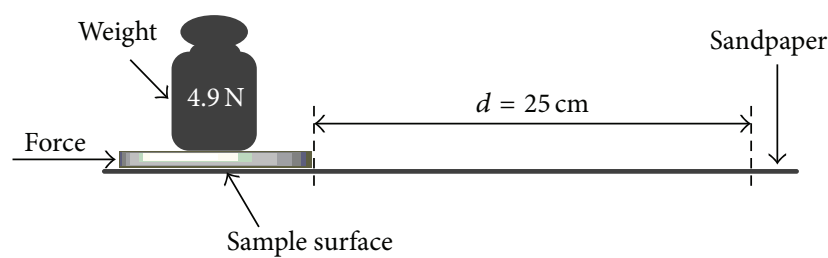

FigURE 1: Schematic representation of abrasion measurement employed to evaluate wearability of the as-prepared material surface.

to fabricate superhydrophobic materials with stable antiwear property.

\section{Experimental Section}

2.1. Materials. The nanoscale silicon dioxide particles $(50 \mathrm{~nm})$ were purchased from Ross Technology Corporation. The microscale silicon dioxide particles $(0.5-10 \mu \mathrm{m}$, approximately $80 \%$ between 1 and $5 \mu \mathrm{m}$ ), the triethoxymethylsilane, and the diethoxydimethylsilane were all purchased from Sigma-Aldrich. Hydrochloric acid ( $\mathrm{HCl}, 37 \%)$, the isopropanol, acetone, and methanol were analytical and used as received without any purification. In all the process, deionized water was used.

\subsection{Fabrication of the Antiwear Superhydrophobic Mate-} rials. The $0.12 \mathrm{~g}$ nanoscale silicon dioxide particles and $0.6 \mathrm{~g}$ microscale silicon dioxide particles were added to $8 \mathrm{~mL}$ mixed solution of isopropanol and water $(\mathrm{V}: \mathrm{V}=$ $2: 1$ ). The mixture was ultrasonic and dispersed for $30 \mathrm{~min}$. Then $0.4 \mathrm{~mL}$ hydrochloric acid, $3.0 \mathrm{~mL}$ triethoxymethylsilane $(0.015 \mathrm{mmol})$, and $0.90 \mathrm{~mL}$ diethoxydimethylsilane $(0.005 \mathrm{mmol})$ were added to the mixed solution. The reaction mixture was stirred for 3 hours at $60^{\circ} \mathrm{C}$. After the mixture was cooled to room temperature, the mixture was then dropped onto the glass slide $(2.5 \mathrm{~cm} \times 2.5 \mathrm{~cm})$ surface until it spread all over the glass surface. A Petri dish cover was placed above the glass in order that the solvent evaporates slowly. After the mixture was dried overnight the samples were heat-treated at $160^{\circ} \mathrm{C}$ for 2 hours.

2.3. Characterization. The methodology illustrated in Figure 1 was invoked to evaluate the antiwear property. The grade of sandpapers adopted here is 180 . The sample surfaces were blowed by the $\mathrm{N}_{2}$ and washed by large amounts of water to remove the abrasion dust after each abrasion test. Contact angles were measured at ambient temperature. The advancing contact angles were measured by advancing a small volume of water onto the surface using a syringe. The receding contact angle was measured by slowly removing water from a drop already on the surface. The water droplets were $5 \mu \mathrm{L}$ for the contact angle measurement and $10 \mu \mathrm{L}$ for the sliding angle for measurement. For each sample, a minimum of four different readings was recorded and the typical error in measurements was $1^{\circ}$. The thickness of the material was measured with an optical microscope by observing the sample's cross section, and three different readings were recorded. The surfaces were observed by the scanning electron microscopy (SEM, XL-30, Philips) to characterize their morphology.

\section{Results and Discussion}

The applications of superhydrophobic surfaces have been greatly hampered by the poor mechanical durability of the microscopic surface topography. Furthermore, most of the artificial superhydrophobic surfaces fabricated with hydrophilic materials required a hydrophobic surface coating and it is obvious that the original hydrophilic materials would be exposed when the surface was mechanically damaged. Herein we adopted the strategy to fabricate the superhydrophobic materials which consist of the microand nanometer scale structures through the whole thickness of materials to resist the abrasion. Two kinds of particles were used for fabricating the micro- and nanometer scale structures which are essential for superhydrophobicity. The triethoxymethylsilane was adopted to polymerize to embed and fix the microparticles. The diethoxydimethylsilane was added to control the polymer with appropriate rigidity, flexibility, and hydrophobicity. After the polymers were cured in oven, the as-prepared particles combined polysiloxane surface has a static water contact angle (CA) of $133^{\circ}$, as shown in Figure 2(a), while the water CA can be greatly increased from $133^{\circ}$ to $160^{\circ}$ after the polysiloxane surface bore a $25 \mathrm{~cm}$ slide on the sandpaper surface.

Although there is no standardized test method to evaluate the mechanical durability of a superhydrophobic surface [19], the apparatus by rubbing the sample against the sandpaper illustrated in Figure 1 was adopted here. Figure 3(a) showed the relationship between the static water CA and the length of the sample surface sliding on sandpaper. The initial water CA of the as-prepared particles combined polysiloxane surface is only $133^{\circ}$, which shows hydrophobicity not superhydrophobicity. After a $25 \mathrm{~cm}$ length rubbing against the sandpaper with a $500 \mathrm{~g}$ weight on the back of the sample, the water contact angle has increased greatly to $160^{\circ}$ and presented the good superhydrophobicity. After one more $25 \mathrm{~cm}$ length slide, there is $2^{\circ}$ increment of water CA. After this the water CA shows little fluctuation and changed from about $162^{\circ}$ to $163^{\circ}$ when the surface bore $75 \mathrm{~cm}$ to $200 \mathrm{~cm}$ length slide abrasion. Figure 2(b) showed the water droplets have a static water $\mathrm{CA}$ of $163^{\circ}$ on the polysiloxane surface which bore a $200 \mathrm{~cm}$ slide on the sandpaper surface. A superhydrophobic steel surface, which is prepared according to Zhang's procedure [21], also underwent the same abrasion test. The result showed that the superhydrophobic steel surface, which is fabricated with hydrophilic material and hydrophobic coatings, has a poor antiwear property. The water CA decreased dramatically from $159^{\circ}$ to $72^{\circ}$ after the superhydrophobic surface endured a $25 \mathrm{~cm}$ length rubbing against the sandpaper with a $500 \mathrm{~g}$ weight on the back of the sample, as shown in Figure 3(a). After one more $25 \mathrm{~cm}$ length slide, the initial superhydrophobic steel surface lost completely its superhydrophobicity and switched to a hydrophilic surface with a water CA of $15^{\circ}$. The thickness 


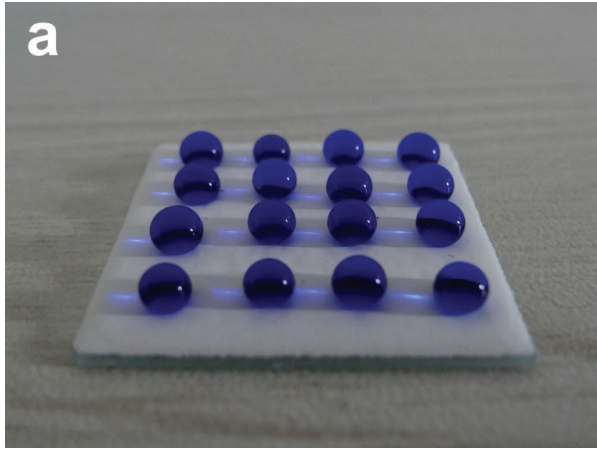

(a)

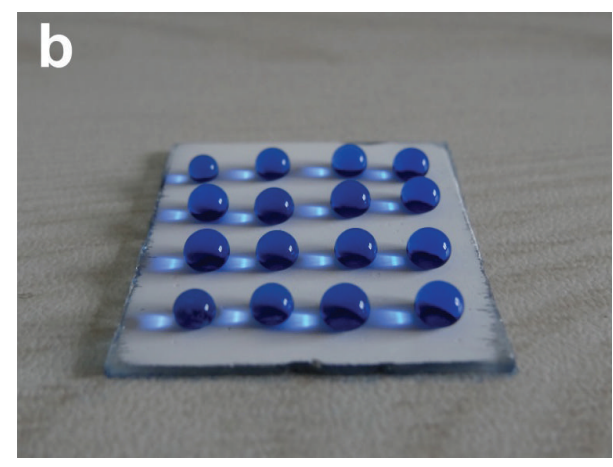

(b)

FIGURE 2: Images of water droplets dyed by methylene blue with different sizes on the particles combined polysiloxane surfaces of the same sample before (a) and after (b) the surface abrasion. The contact angles of the water droplets on the corresponding surfaces are $133^{\circ}$ (a) and $163^{\circ}$ (b), respectively.

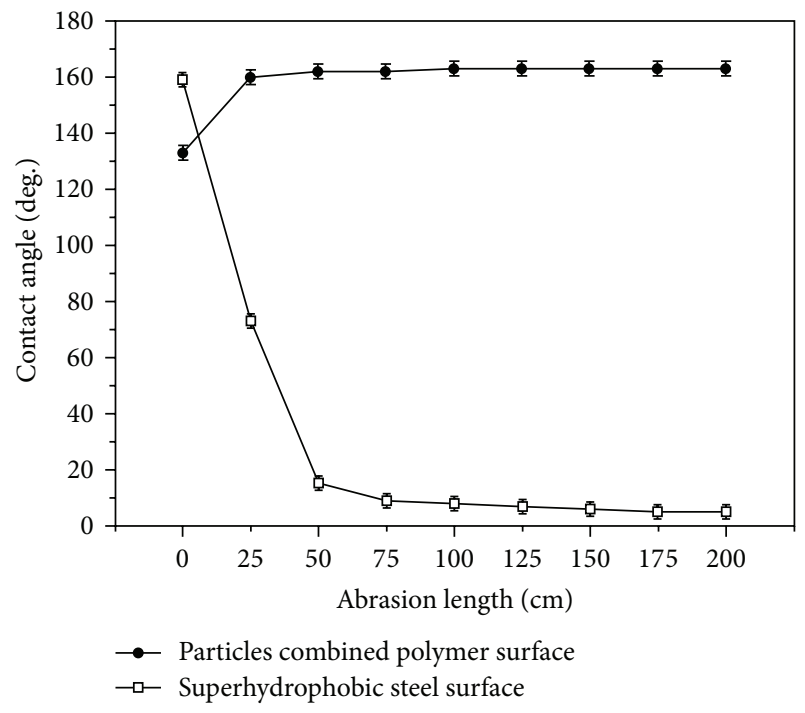

(a)

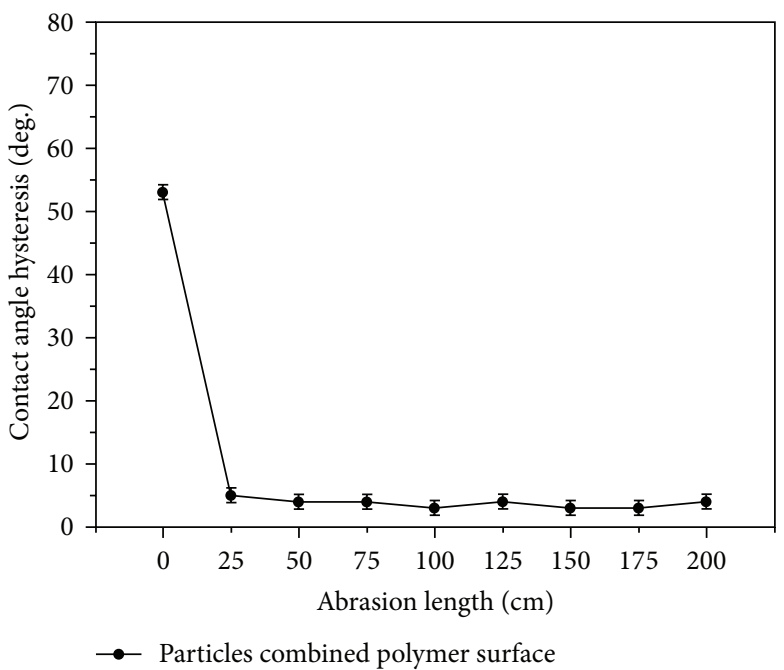

(b)

FIGURE 3: (a) The relationship between the static water contact angle and the lengths of the as-prepared polysiloxane surface (superhydrophobic steel surface) sliding on the sandpaper. (b) The relationship between the contact angle hysteresis and the lengths of the as-prepared polysiloxane surface sliding on the sandpaper.

measurements have also been carried out with an optical microscope before and after the abrasion test to investigate the thickness change of the polysiloxane film. It showed that the polysiloxane film of the as-prepared sample is $0.89 \mathrm{~mm}$, while the thickness of the film decreased to $0.22 \mathrm{~mm}$ after the surface bore the $200 \mathrm{~cm}$ length slide abrasion. These results clearly revealed that the water repellency of the particles combined polysiloxane film not only has the mechanical durability but also can be enhanced with the increase of surface roughness by the surface abrasion.

In addition to a high static contact angle, a small contact angle hysteresis is also vital for a surface to be truly superhydrophobic because a small hysteresis leads to a water droplet being able to roll off of a surface easily. Thus, the contact angle hysteresis after the sample rubbed against the sandpaper should also be measured. Figure 3(b) showed the CA hysteresis of the as-prepared particles combined polymers after each slide. Before the surface abrasion, the initial combined polysiloxane surface has a CA hysteresis of $53^{\circ}$, which is relatively high and implying a high roll-off angle. After a $25 \mathrm{~cm}$ slide against the sandpaper, the CA hysteresis has decreased greatly to $5^{\circ}$ and the falling water droplet already can bounce from the surfaces and eventually roll off without ever coming to rest on the surface. The CA hysteresis shows little fluctuation when the surface bore $75 \mathrm{~cm}$ to $200 \mathrm{~cm}$ length slide abrasion. These results clearly proved that the asprepared particles combined polymers have remained of the true superhydrophobicity even after a severe surface abrasion.

The initial as-prepared polysiloxane surface has a water CA of $133^{\circ}$, while the water CA is increased greatly to $162^{\circ}$ after the surface abrasion. The great changes of the surfaces morphology before and after the surface abrasion have been 


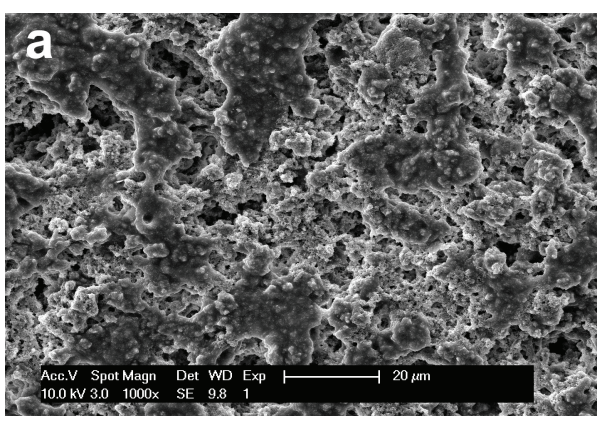

(a)

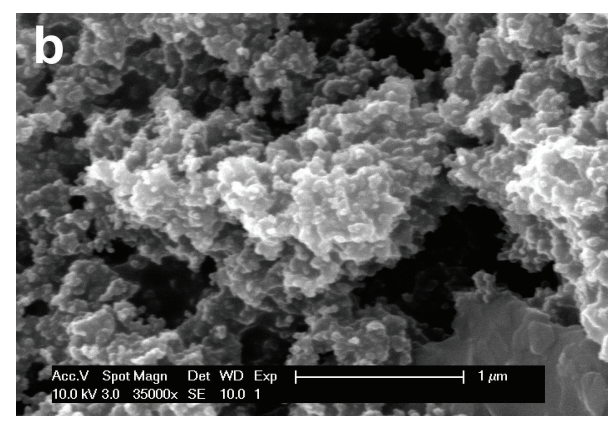

(b)

FIGURE 4: (a) SEM images of the as-prepared particles combined polysiloxane surface before the abrasion. (b) The porous and rough structures with micro- and nanometer scale exist below the outmost surface. The scale bars represent $20 \mu \mathrm{m}$ (a) and $1 \mu \mathrm{m}$ (b), respectively.

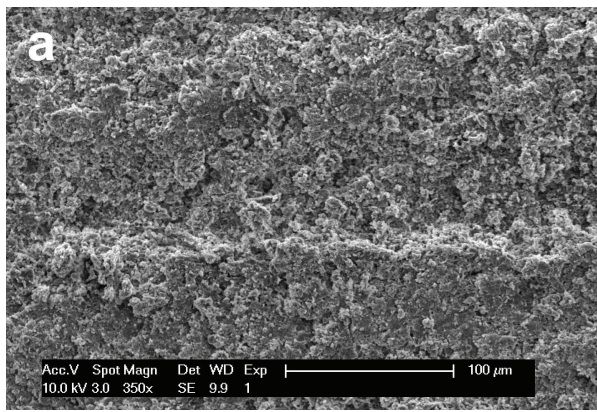

(a)

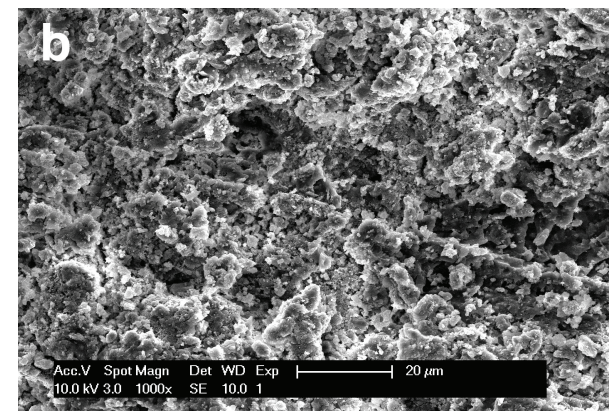

(b)

FIGURE 5: SEM images at different magnifications of the particles combined polysiloxane surface after the abrasion. The scale bars represent $100 \mu \mathrm{m}$ (a) and $20 \mu \mathrm{m}$ (b), respectively.

observed carefully by the scanning electron microscopy. Figure 4 is the SEM images before the surface abrasion test. There is a porous and rough structure which made up the materials. However, many-large-island structure with diameters of about $20-50 \mu \mathrm{m}$ is distributed randomly across the upper surface, as shown in Figure 4(a). Figure 4(a) also revealed that the top of the island structure is relatively flat and there is no porous structure on the island surfaces. This relative flat surface probably formed during the process of the solvent evaporate process has decreased the surface roughness to a certain extent and lowered the water CA as a result. According to the Cassie equation [22],

$$
\cos \theta_{r}=f_{1} \cos \theta-f_{2},
$$

which is generally valid for heterogeneous surfaces, composed of air and a solid with superhydrophobicity. Here, $\theta_{r}$ $\left(132^{\circ}\right)$ is the CA of the initial particles combined polysiloxane surface. $\theta\left(107^{\circ}\right)$ is the CA of the smooth polysiloxane surface without particles and $f_{1}$ and $f_{2}$ are the fractional interfacial areas of the microstructures and of the air in the interspaces among the microstructures, respectively (i.e., $\left.f_{1}+f_{2}=1\right)$. According to the equation, the $f_{2}$ value of the rough surface with the microstructures is estimated to be 0.53 . This means that air occupies only $53 \%$ of contact area between the water droplet and the microstructures. Figure $4(\mathrm{~b})$ is a magnified image of the porous and rough structures with micrometer scale existing below this flat island. Figure 4(b) showed that the nanoscale particles are distributed across the rough structure surface. These microand nanometer scale structures inside the surface have the certain superhydrophobicity and it was exposed after the top flat islands were gotten rid of.

Figure 5(a) is the low-magnification image of the new surface produced by the surface abrasion. Compared with Figure 4(a), the main difference is that there are no more island structures on the top of surface and the remarkable scratches appeared across the surface. Figure 5(b) showed that a furrow-like structure with width of $20-50 \mu \mathrm{m}$ micrometer was parallel distributed on the surface. These scratches which are produced by the sandpaper during sample sliding on sandpaper surface are vital to the superhydrophobicity since this behavior has gotten rid of the flat-top island and greatly increased the surface roughness. These images clearly revealed that a new surface appeared after the surface abrasion just like the natural superhydrophobic plant leaves regenerating their hydrophobic wax coating. The existence of the nanometer particles is also important to the superhydrophobicity for forming the nanometer scale structure and bestowing the synergistic binary geometric structures on the surfaces. These images demonstrated that the particles combined polymers surface after the surface abrasion has the structures at the micro- and nanometer scale, and such synergistic binary geometric structures made the surface 
roughness increase and the air fraction of the interfacial areas high enough. The binary micro- and nanometer scale structures have already trapped enough amount of air to prevent the penetration of the water droplet into the holes, which bestowed the superhydrophobicity on the surfaces. According to the Cassie equation, the $f_{2}$ value of the rough surface with the hierarchical rough structures is estimated to be 0.93 . This means that air already occupies $93 \%$ of contact area, which is responsible for the enhancement of superhydrophobicity after the surface abrasion.

\section{Conclusions}

In summary, we fabricated the mechanically durable superhydrophobic surfaces with organic silane and particles by polymerizing silane and embedding the particles. The asprepared particles combined polysiloxane surface showed stable superhydrophobicity even after the surface underwent a long distance friction. Furthermore, the superhydrophobicity of the polymer materials can be enhanced or restored. These properties will greatly accelerate and broaden the applications of superhydrophobic surfaces. We expect this strategy will open a new avenue in the preparation of mechanically durable superhydrophobic surface.

\section{Conflict of Interests}

The authors declare that there is no conflict of interests regarding the publication of this paper.

\section{Acknowledgments}

The authors thank the National Natural Science Foundation of China (Grants no. 21473132 and no. 21102113), the Natural Science Foundation of Shaanxi Province, China (Grant no. 2014JM2047), and the Natural Science Research Project of Science and Technology Agency of Shaanxi Province, China (Grant no. 2013KJXX-41), for continuing financial support.

\section{References}

[1] T. Sun, L. Feng, X. Gao, and L. Jiang, "Bioinspired surfaces with special wettability," Accounts of Chemical Research, vol. 38, pp. 644-652, 2005.

[2] W. Barthlott and C. Neinhuis, "Purity of the sacred lotus, or escape from contamination in biological surfaces," Planta, vol. 202, no. 1, pp. 1-8, 1997.

[3] L. Passoni, G. Bonvini, A. Luzio, A. Facibeni, C. E. Bottani, and F. Di Fonzo, "Multiscale effect of hierarchical self-assembled nanostructures on superhydrophobic surface," Langmuir, vol. 30, no. 45, pp. 13581-13587, 2014.

[4] J. C. Tuberquia, N. Nizamidin, R. R. Harl et al., "Surfaceinitiated polymerization of superhydrophobic polymethylene," Journal of the American Chemical Society, vol. 132, no. 16, pp. 5725-5734, 2010.

[5] S. Huang, "Mussel-inspired one-step copolymerization to engineer hierarchically structured surface with superhydrophobic properties for removing oil from water," ACS Applied Materials \& Interfaces, vol. 6, no. 19, pp. 17144-17150, 2014.
[6] N. Verplanck, E. Galopin, J.-C. Camart, V. Thomy, Y. Coffinier, and R. Boukherroub, "Reversible electrowetting on superhydrophobic silicon nanowires," Nano Letters, vol. 7, no. 3, pp. 813817, 2007.

[7] T. Sun, G. Wang, H. Liu, L. Feng, L. Jiang, and D. Zhu, "Control over the wettability of an aligned carbon nanotube film," Journal of the American Chemical Society, vol. 125, no. 49, pp. 1499614997, 2003.

[8] J. Bravo, L. Zhai, Z. Wu, R. E. Cohen, and M. F. Rubner, "Transparent superhydrophobic films based on silica nanoparticles," Langmuir, vol. 23, no. 13, pp. 7293-7298, 2007.

[9] K. Tadanaga, J. Morinaga, A. Matsuda, and T. Minami, "Superhydrophobic-superhydrophilic micropatterning on flowerlike alumina coating film by the sol-gel method," Chemistry of Materials, vol. 12, no. 3, pp. 590-592, 2000.

[10] Y. Li, X. J. Huang, S. H. Heo et al., "Superhydrophobic bionic surfaces with hierarchical microsphere/SWCNT composite arrays," Langmuir, vol. 23, no. 4, pp. 2169-2174, 2007.

[11] I. P. Parkin and R. G. Palgrave, "Self-cleaning coatings," Journal of Materials Chemistry, vol. 15, no. 17, pp. 1689-1695, 2005.

[12] L. Cao, A. K. Jones, V. K. Sikka, J. Wu, and D. Gao, "Antiicing superhydrophobic coatings," Langmuir, vol. 25, no. 21, pp. 12444-12448, 2009.

[13] J. A. Howarter and J. P. Youngblood, "Self-cleaning and antifog surfaces via stimuli-responsive polymer brushes," Advanced Materials, vol. 19, no. 22, pp. 3838-3843, 2007.

[14] X. Gao, X. Yan, X. Yao et al., “The dry-style antifogging properties of mosquito compound eyes and artificial analogues prepared by soft lithography," Advanced Materials, vol. 19, no. 17, pp. 2213-2217, 2007.

[15] L. Gao and T. J. McCarthy, “"Artificial lotus leaf” prepared using a 1945 patent and a commercial textile," Langmuir, vol. 22, no. 14, pp. 5998-6000, 2006.

[16] W. Choi, A. Tuteja, S. Chhatre, J. M. Mabry, R. E. Cohen, and G. H. McKinley, "Fabrics with tunable oleophobicity," Advanced Materials, vol. 21, no. 21, pp. 2190-2195, 2009.

[17] Q. Pan and M. Wang, "Miniature boats with striking loading capacity fabricated from superhydrophobic copper meshes," ACS Applied Materials \& Interfaces, vol. 1, no. 2, pp. 420-423, 2009.

[18] J. Ou, B. Perot, and J. P. Rothstein, "Laminar drag reduction in microchannels using ultrahydrophobic surfaces," Physics of Fluids, vol. 16, no. 12, pp. 4635-4643, 2004.

[19] T. Verho, C. Bower, P. Andrew, S. Franssila, O. Ikkala, and R. H. A. Ras, "Mechanically durable superhydrophobic surfaces," Advanced Materials, vol. 23, no. 5, pp. 673-678, 2011.

[20] Y. Li, L. Li, and J. Sun, "Bioinspired self-healing superhydrophobic coatings," Angewandte Chemie, vol. 49, no. 35, pp. 6129-6133, 2010.

[21] M. Qu, B. Zhang, S. Song, L. Chen, J. Zhang, and X. Cao, "Fabrication of superhydrophobic surfaces on engineering materials by a solution-immersion process," Advanced Functional Materials, vol. 17, no. 4, pp. 593-596, 2007.

[22] A. B. D. Cassie and S. Baxter, "Wettability of porous surfaces," Transactions of the Faraday Society, vol. 40, pp. 546-551, 1944. 

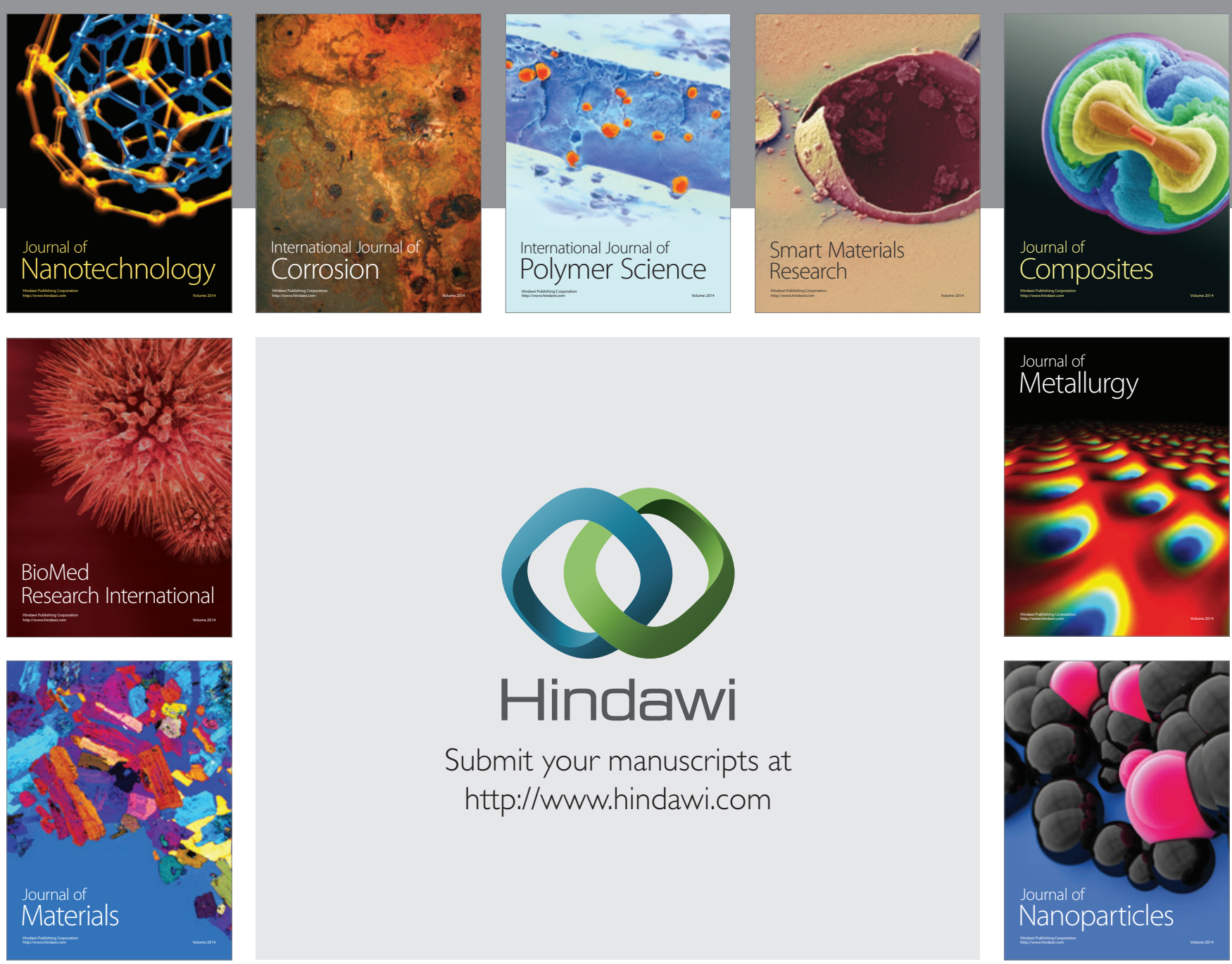

Submit your manuscripts at http://www.hindawi.com
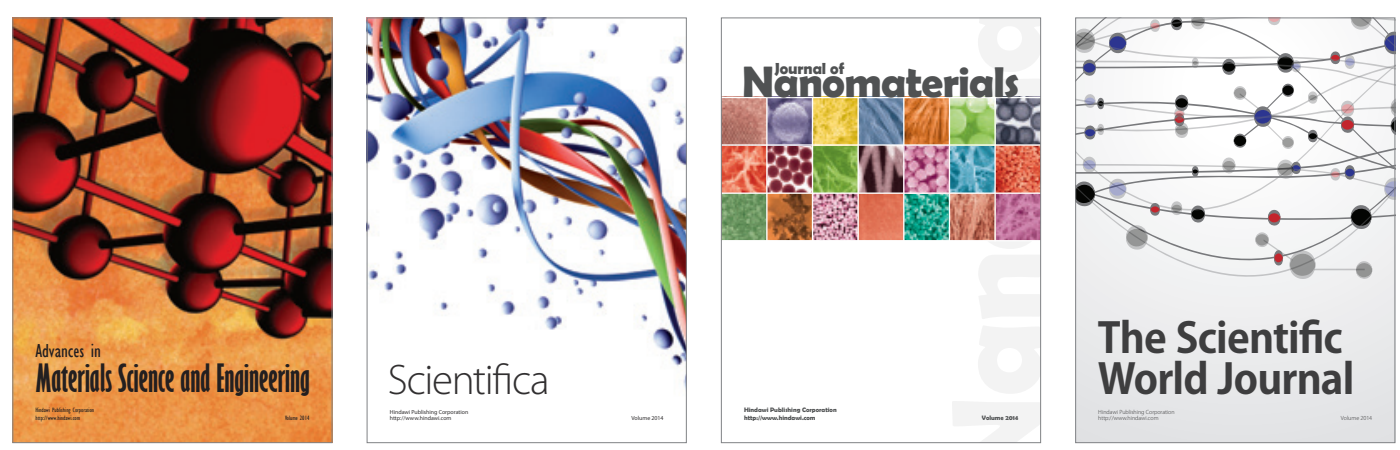

\section{The Scientific World Journal}
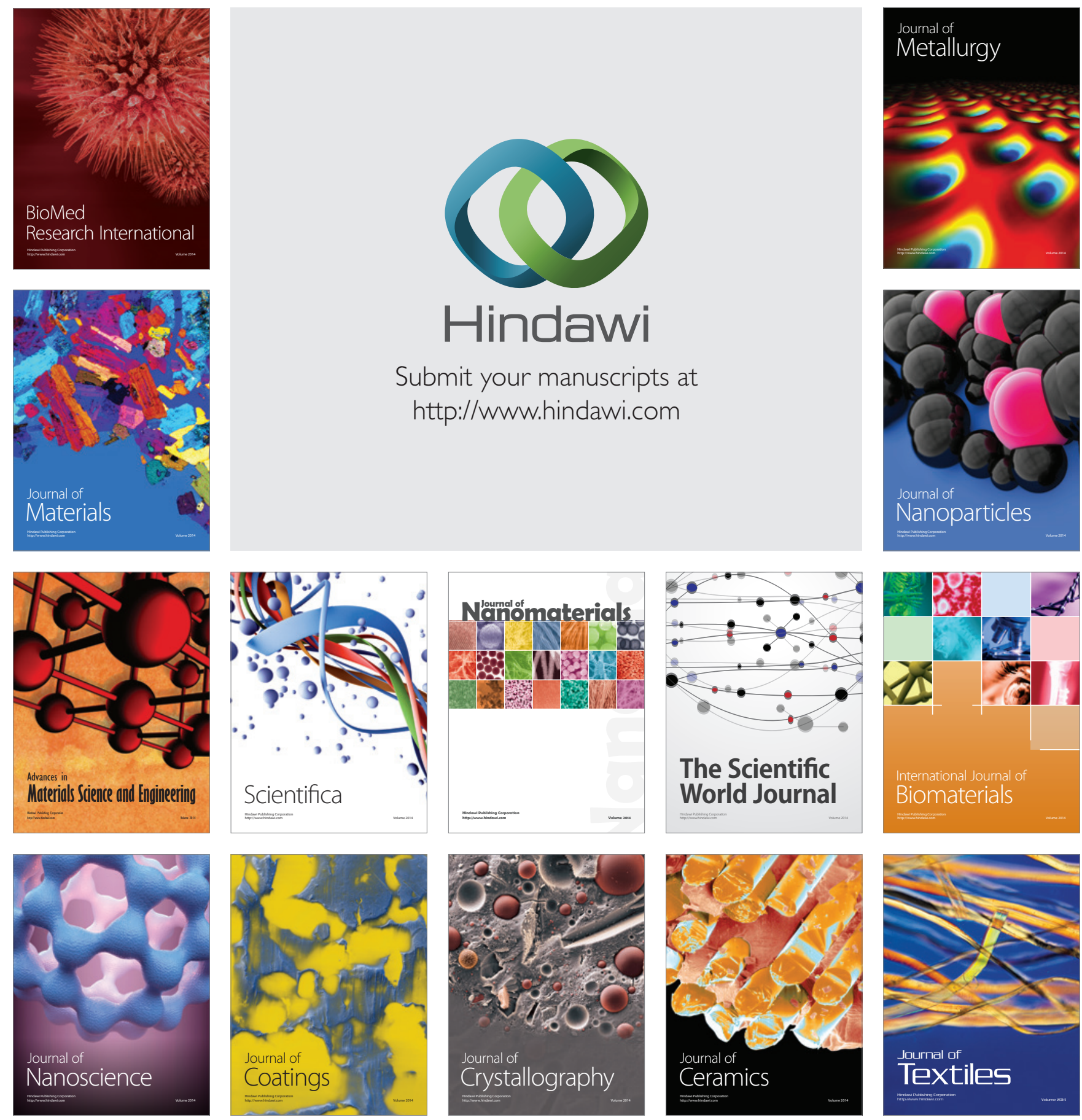